\title{
Incomplete, Incorrect or Alternative Diagnosis Code Assigned
}

National Cancer Institute

\section{Source}

National Cancer Institute. Incomplete, Incorrect or Alternative Diagnosis Code Assigned. NCl Thesaurus. Code C153580.

A diagnosis code was assigned based on incomplete, incorrect or non-ideal (an alternative diagnosis may be more appropriate) supporting information. 